\title{
Clinical Management of Organophosphate Poisoning in an Indian Spectacled Cobra (Naja naja; Linnaeus, 1758)
}

\author{
C. M. Bhadesiya*, G. R. Chaudhary, T. Patel, V. A. Patel, M. Anikar and P. J. Gajjar \\ Postgraduate Institute of Veterinary Education \& Research (PGIVER) \\ Kamdhenu University. Rajpur (Nava), Himmatnagar-383010, Gujarat (India) \\ *Corresponding author
}

\section{A B S T R A C T}

\section{Keywords}

Organophosphate Poisoning, Indian spectacled cobra, Clinical signs,

Therapeutic management

Article Info

Accepted:

18 May 2020

Available Online:

10 June 2020
Organophosphate compounds are commonly used in agricultural fields to control insects and pests. The Indian spectacled cobra (Naja naja; Linnaeus, 1758) is one of the four major venomous snakes found on different terrains in Gujarat, India. Diagnosis and therapeutic management of clinical diseases of Indian spectacled cobra are not published at length. The present report documents the first record of accidental poisoning by organophosphate in an Indian spectacled cobra along with elucidation on clinical signs and successful therapeutic management.

\section{Introduction}

Organophosphate compounds (OPCs) are commonly used in field to control insects and parasites in plants and animals (e.g., as an insecticide/pesticide, acaricide, pour-on, shipdip etc.). OPCs can be absorbed by dermal absorption, ingestion and inhalation following an accidental exposure. They are known to cause poisoning with variable symptoms in humans and domestic animals such as cattle (Khan et al., 1961; Khan, 2001; Naik et al., 2011) and dogs (Ola-Davis et al., 2018;
Asokan et al., 2019). OPC poisoning is not documented in wild reptiles.

The Indian spectacled cobra (Naja naja; Linnaeus, 1758), a reptile, is commonly found in forests, rocky terrains, agricultural fields in and around urban and rural areas. Detailed description on clinical symptoms, diagnosis, therapeutic management, prevention and control of common diseases of Indian spectacled cobra is not available. Veterinarians and academicians have made frequent efforts to document a few ailments 
such as necrosed venom gland (Raj et al., 2017), injury (Raut et al., 2008), tick infestation (Catherine et al., 2017) etc. which are available in form of individual case reports or studies conducted in captive facilities. To the authors' knowledge, there is no known published report on organophosphate (OP) poisoning in an Indian spectacled cobra.

The present report documents first record of OP poisoning in an Indian spectacled cobra in Gujarat, India. Emphasis has been placed specifically on clinical symptoms, applicable restraint method and clinical management of the case for successful outcome.

\section{Case history and clinical observations}

An Indian spectacled cobra was rescued from an agricultural field and brought to the veterinary hospital functioning under the Postgraduate Institute of Veterinary Education \& Research (PGIVER), Kamdhenu University, Rajpur (Nava), Himmatnagar, Gujarat for treatment. It was found to have sluggish response to external stimuli and reluctant movement at the time of rescue. History revealed recent use of OPC at the place of rescue. No other specific details could be extracted through the anamnesis.

The Indian spectacled cobra was restrained physically by securing its head in a piece of commonly used pipe (Fig.1(a)). A detailed physical examination revealed clinical signs such as (1) slightly open mouth with frequent protrusion of tongue (Fig.1(b)); (2) rough skin surface/scales; (3) presence of dried powdery material on external surface of head (Fig.1(b)) and on scales over the body; (4) moderate miosis (Fig.1(b)); (5) dullness; (6) depression; (7) lethargy; (8) sluggish movements; (9) lacrimation; (10) absence of ectoparasite; (11) no evidence of traumatic injuries; (12) no evidence of major blood loss; (13) no abnormal discharge of presence of blood spots on external orifices; (14) no removal of excreta while handling; (15) reduced response to external stimuli; (16) no quick attempts for self-protection when approached for physical restraint; (17) spasms on muscular parts of the body; and (18) infrequent jerky movements from caudal $2 / 3^{\text {rd }}$ part of the body.

\section{Treatment and Discussion}

Reptiles generally remove excreta when they are restrained by physical methods. They also show protective behavior when approached for the physical restraint. Such observations were not found in the present case which enabled the handler for proper use of physical method of restraint. A piece of commonly used pipe was used to secure the head while remaining part of the body was kept inside the jar in which it was brought to the hospital. Care was taken to keep one end of the pipe open to allow breathing, to perform lavage and to perform oral administration of medication. This restraint method reduced chances of accidental snake-bite to handler and veterinarian during treatment. The therapeutic management of the case included following major aspects.

\section{Gastric lavage and Use of lukewarm water}

Efforts were made to perform gastric lavage by use of sterilized sheath which is commonly used to perform artificial insemination in large ruminants. One end of the sheath was devoid of sharp edges and care was taken to keep the anterior $1 / 3^{\text {rd }}$ part of body in straight and stable position. These considerations prevented common harmful effects such as infection and damage to internal organs during lavage. Ingested material or prey was not found after completion of gastric lavage. This observation indicated that OP poisoning in the present case could have been caused by accidental dermal exposure and not by 
ingestion of a prey which could have died of OPC at place of rescue. In general, dermal absorption of any agent is slower. Therefore, the entire body was cleaned and submerged in lukewarm water for some time to remove OPC. Care was taken to keep the head in a secured position to prevent aspiration of water into the lungs.

\section{Oral administration of vitamins}

Oral administration of a preparation containing combination of vitamin-A, E, B 12 and $\mathrm{D}_{3}$ was performed (Fig.2). Hypothesis behind use of this preparation was generated on the basis of effects of vitamins. Vitamin-A is essential for health of epithelial cells, vitamin-E is known to have antioxidant properties and beneficial effects on muscles, vitamin- $\mathrm{B}_{12}$ is a nutrient important for nerve while vitamin- $\mathrm{D}_{3}$ is important for calcium metabolism. Such a combination often reduces stress which is necessary for successful outcome in critical cases.

\section{Specific antidote}

There is no published report on availability of specific antidote for OP poisoning in an Indian spectacled cobra. The OPCs are known to inhibit acetylcholinesterase (AChE) which is an important enzyme for humans, some animals and insects. Therefore use of anticholinergic drug such as atropine can be used for treatment. Khan (2001) and Naik et al. (2011) have documented use of atropine sulfate during treatment of OP poisoning in cattle. In the present case, the Indian spectacled cobra was injected with atropine sulfate within the limits of recommended dose rate $(0.01-0.02 \mathrm{mg} / \mathrm{kg})$ intramuscularly (Fig.3).

\section{Post-treatment observation and release}

Post-treatment observation was carried out to evaluate clinical status of the Indian spectacled cobra for a few hours. The cobra was placed back into the jar. The jar was kept in a dark place for few hours. Efforts were made to avoid any disturbance. The cobra was found to have gradual improvement in activity and movement after one hour of treatment. It started reacting normally to external stimuli and recovered uneventfully after two hours of treatment. Later, advice was given to the rescuer to release the Indian spectacled cobra in a suitable place away from human activities (Fig.4). Timely release is believed to improve chances of survival because snakes often retain their normal behavior.
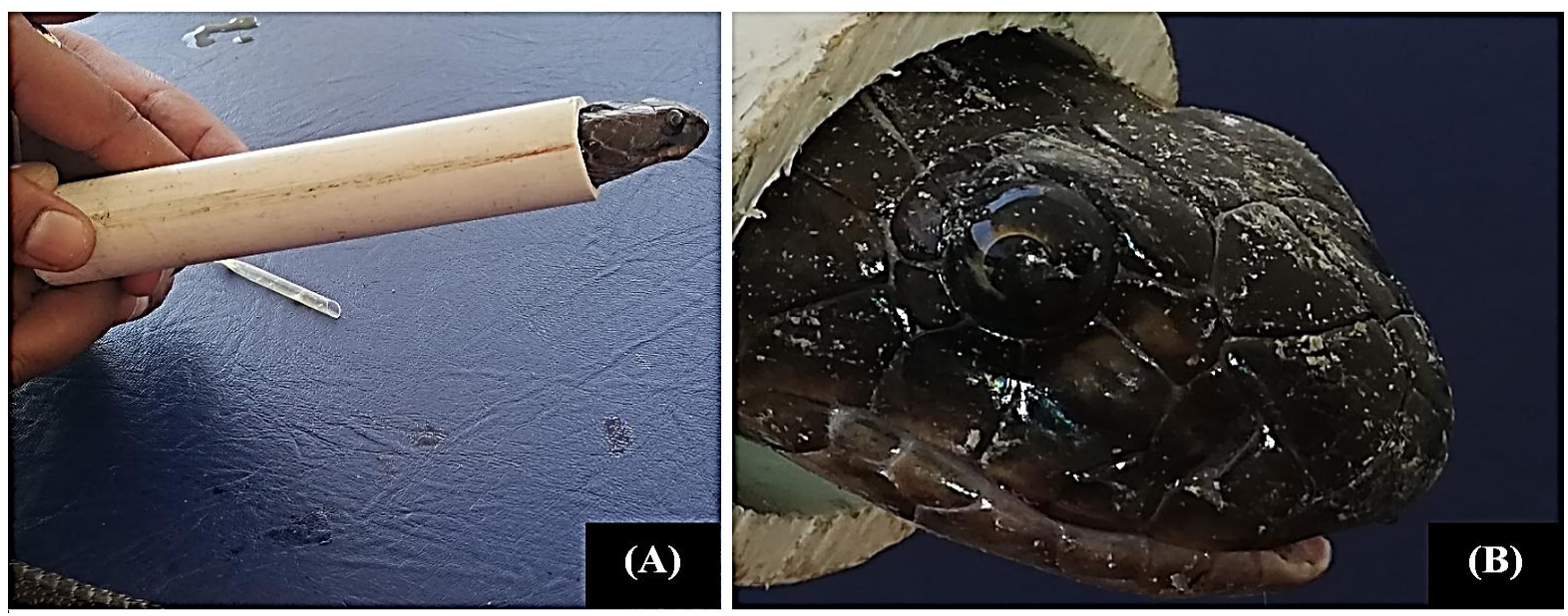

Fig.1 (A) Use of pipe to secure the head of Indian spectacled cobra; (B) Slightly open mouth, moderate miosis and presence of dried powdery material on head region 


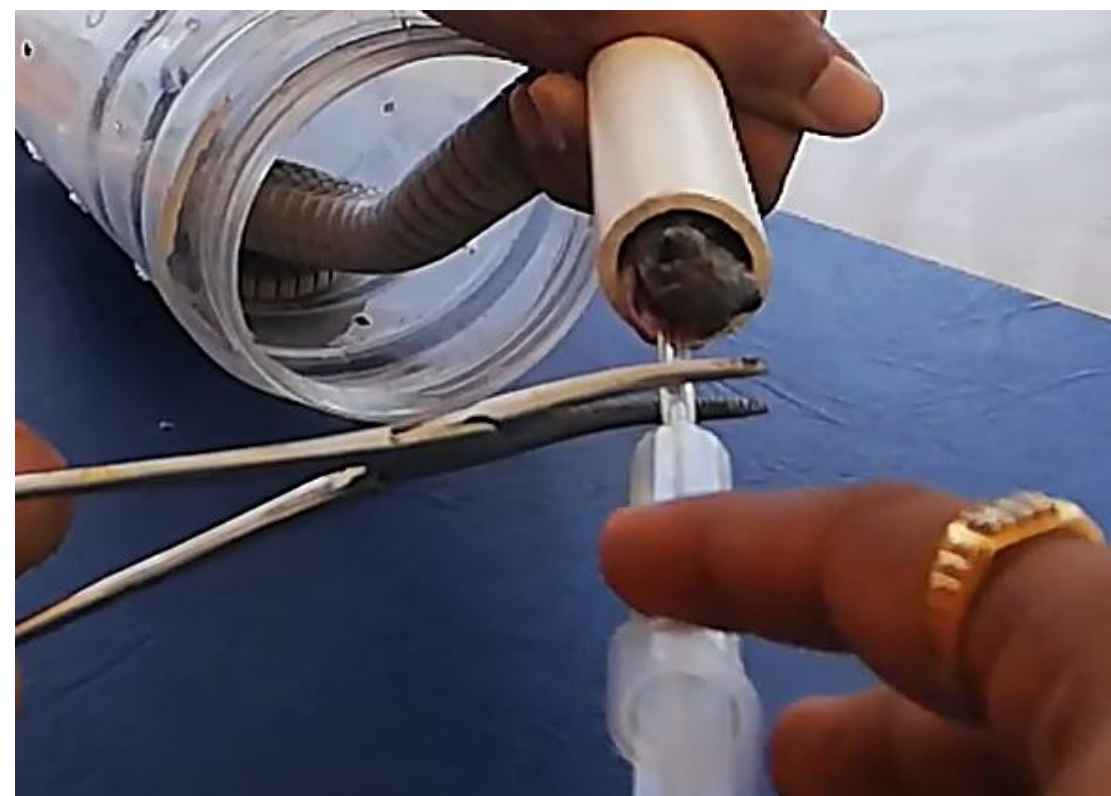

Fig.2 Oral administration of a preparation containing vitamins after proper physical restraint

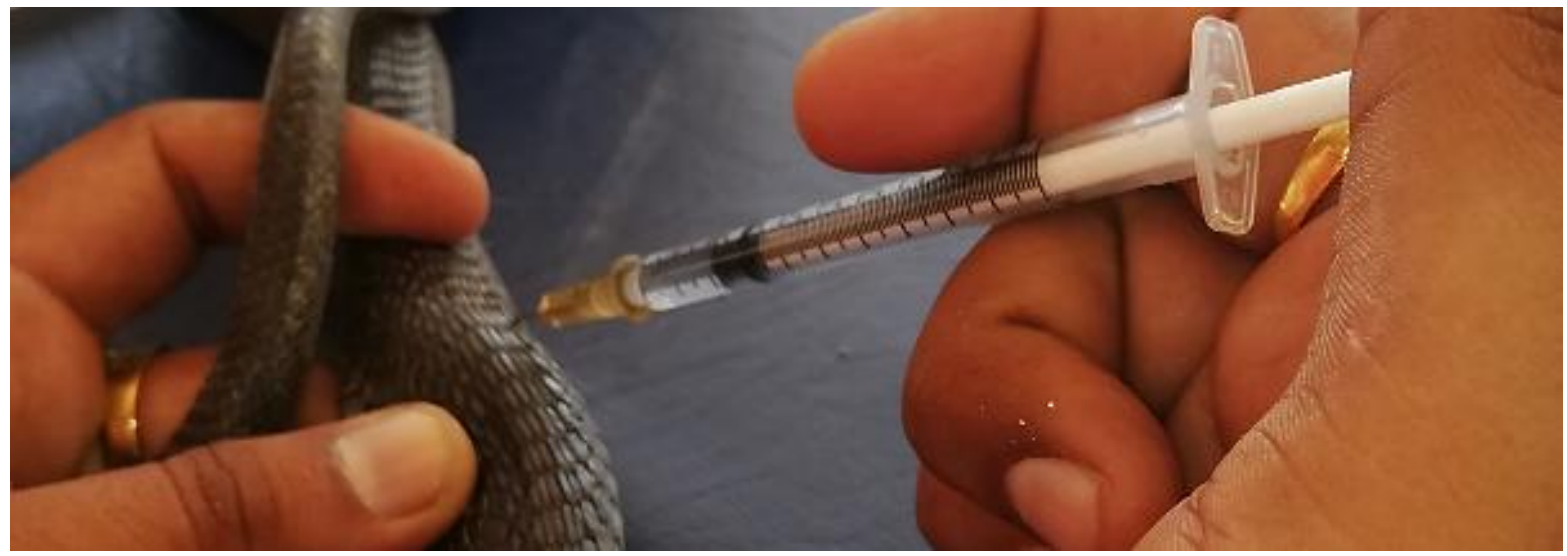

Fig.3 Intramuscular injection of atropine sulfate

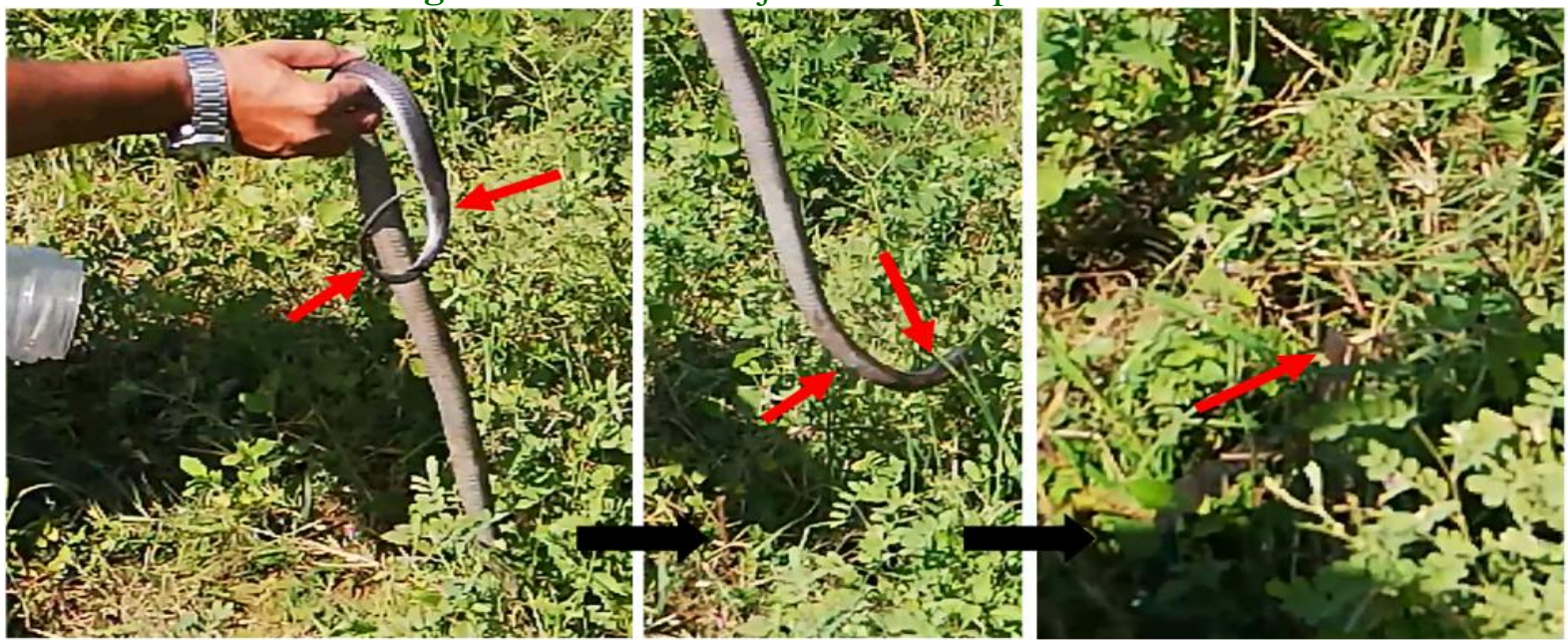

Fig.4 Successful and safe release in a suitable area away from human activities (Red arrows: Improvement in bodily movements at the time of release) 
The extent of deforestation, habitat loss and increased human interference has resulted in close propinquity of Indian spectacled cobra to humans. This leaves a potential chance of rise in known or unknown and accidental or intentional conflicts. Indian spectacled cobra can also suffer from naturally occurring diseases. Therefore, it is necessary to document individual disease conditions to generate a strong database for future research in veterinary science.

In conclusion, first record of OP poisoning and its therapeutic management in an Indian spectacled cobra has been documented. Proper method of physical restraint, careful observation of clinical signs and selection of appropriate therapeutic agents resulted in successful recovery.

\section{Conflict of Interest \& Acknowledgement}

Authors declare no conflict of interest with regards to funding. Authors acknowledge members of the Himmatnagar Nature Club (HNC), staff of regional forest department and staff of veterinary hospital.

\section{References}

Asokan, V.R., Kerl, M.E., Evans, T. and Harmon, M. (2019). Organophosphate intoxication in 2 dogs from ingestion of cattle ear tags. J. Vet. Emerg. Crit. Care (San Antonio), 29(4):424-430.
Catherine, B.R., Jayathangaraj, M.G., Soundararajan, C., Guru, B. and Yogaraja, D (2017). Prevalence of Amblyomma gervaisi ticks on captive snakes in Tamil Nadu. J. Parasit. Dis., 41(4):952-958.

Khan, M.A., Kramer, T. and Avery, R. J. (1961). Organophosphate poisoning in cattle with particular reference to $\mathrm{Co}$ Ral. Can. Vet. J., 2(6):207-211.

Khan, O. (2001). Organophosphate poisoning in a group of replacement heifers and dry cows. Can. Vet. J., 42:561-563.

Naik, G. S., Kotresh, A.M., Shambulingappa, B.E. and Ananda, K.J. (2011). Organophosphorus compound poisoning in cross bred cows - a case report. Vet. World, 4(2):88.

Ola-Davis, O.E., Azeez, O.I., Oyagbemi, A.A. and Abatan, M.O. (2018). Acute coumaphos organophosphate exposure in the domestic dogs: Its implication on hematology and liver functions. Int. J. Vet. Sc. Med., 6(1):103-112.

Raj, R., Mukherjee, P., Chaudhari, S., Basu, S. and Datta, U. (2017). Surgical removal of necrosed venom gland in Indian spectacled cobra (Naja naja) - a case report. Bulletin UASVM Veterinary Medicine 74(1):126-128.

Raut, P.A., Sonkhusale, V.G., Khan, L.A., Nakade, M.K. and Bodkhe, A.M. (2008). A case report of management of snake's injury in captivity. Vet. World, 1(11):346.

\section{How to cite this article:}

Bhadesiya, C. M., G. R. Chaudhary, T. Patel, V. A. Patel, M. Anikar and Gajjar, P. J. 2020. Clinical Management of Organophosphate Poisoning in an Indian Spectacled Cobra (Naja naja; Linnaeus, 1758). Int.J.Curr.Microbiol.App.Sci. 9(06): 2356-2360.

doi: https://doi.org/10.20546/ijcmas.2020.906.288 